\title{
Discussion
}

on paper published in

Advances in Cement Research

1994, 6, No. 24, Oct., 147-154

\section{The fractal surface of cementitious materials determined by impedance spectroscopy}

\author{
W. J. McCarter
}

\author{
Contribution by S. W. Massey \\ Materials Division, Building Research Establishment, Garston, Watford, UK
}

The paper by McCarter describes the use of impedance spectroscopy to determine the fractal dimension of cement. Measurements on a variety of sedimentary rocks are cited as evidence for fractal pore structures in porous materials $s^{1-5}$ and as partial validation of the measurements on cementitious material cited in the paper. However, there are some distinct differences between the pore structures of sedimentary rocks and cement pastes. The primary difference is that the pore space in sedimentary rock is fractal by nature of its construction, which involves the accumulation of material. ${ }^{3}$ It has been shown that granites, which form by crystal growth, do not have fractal pore spaces. ${ }^{6}$ This is much more akin to the growth of phases that occur in the hardening of cement. Nevertheless, while the pore spaces themselves may not be fractal, the boundaries of the pore space can be formed by fractal surfaces. The distinction as to which part of a geometric object is fractal needs to be clarified regarding the solid phases, boundary surfaces and pore space before results from different materials can be readily transferred. Sedimentary rocks have the distinction of a fractal pore volume and a fractal boundary surface.

Another significant difference between cementitious materials and sedimentary rocks is their response to moisture. For most rocks the internal geometry is fixed irrespective of the moisture content. This is not true for cements, where various gels change shape in response to moisture, hydration and pozzolanic activity. McCarter remarks that the last two activities should have ceased for the samples tested. What effect the moisture response of the gels has on the measurement of the fractal dimension by various methods is largely unknown. However, impedance spectroscopy is very sensitive to moisture content, and the shape of the pores and the micro-fabric govern the volume and distribution of moisture within a material. Thus, the validity of impedance measurements and the power law exponent needs to be explored carefully bearing in mind that moisture changes of the order of $0.001 \%$ (by weight) can be detected in sandstone using impedance spectroscopy (unpublished Building Research Establishment (BRE) report). This is sufficiently accurate to measure the transfer of moisture during changes in the ambient relative humidity levels.

McCarter uses the arguments of Wong ${ }^{7}$ to estimate the characteristic diffusion path $\lambda$. The magnitude of the characteristic diffusion path should depend on the amount of free water in the specimen. Greater amounts of water should result in longer diffusion paths. Experiments on sandstones carried out at the BRE show that the lower frequency limit increases with inreasing moisture content (Table 1), indicating a decrease in the diffusion path length. This seems to suggest that the average drift of ions 


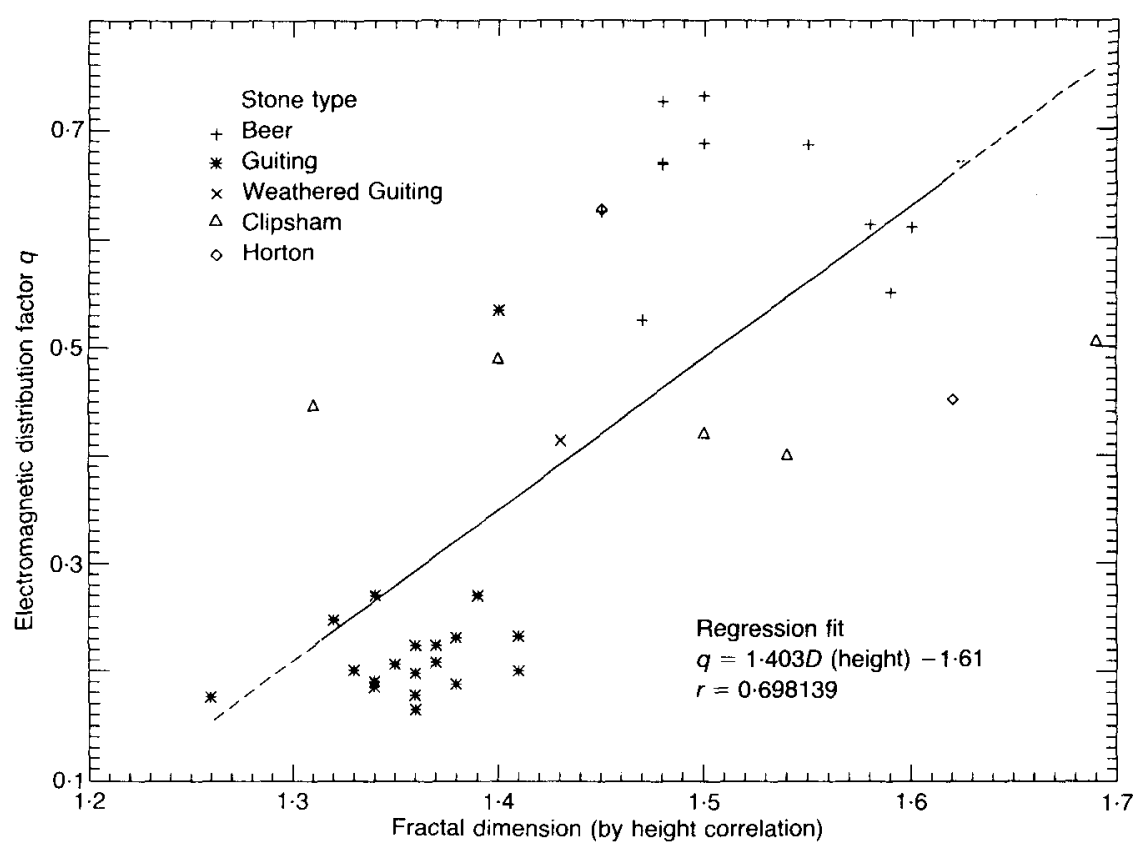

Fig. 1. Fractal dimension plotted against $q$

Table 1. Moisture and diffusion path length

\begin{tabular}{l|c|c}
\hline Moisture: wt \% & $\begin{array}{c}\text { Low-frequency limit: } \\
\mathrm{kHz}\end{array}$ & $\begin{array}{c}\text { Diffusion path length: } \\
\mathrm{m} \times 10^{-7}\end{array}$ \\
\hline $0 \cdot 05$ & $0 \cdot 05$ & $17 \cdot 8$ \\
$0 \cdot 18$ & $1 \cdot 0$ & 3.9 \\
1.13 & $7 \cdot 3$ & 1.4 \\
$2 \cdot 2$ & $5 \cdot 5$ & 0.5 \\
$4 \cdot 3$ & 190 & 0.3 \\
\hline
\end{tabular}

postulated by Wong ${ }^{7}$ is the wrong mechanism for fractal dependance on $q$. However, plots of fractal dimension against $q$ for a number of sedimentary rocks show that the form of the relationship is $D \propto q$ (Fig. 1).

\section{Reply by author}

I wish to thank Dr Massey for his contribution on this paper. Interestingly, Ref. 5 discusses the fractal nature of granite surfaces; however, this reply will be confined to cementitious materials and related issues as raised by Dr Massey.

The fact that cement paste possesses both surface and mass fractals is well documented, ${ }^{8-10}$ with the cement gel comprising $5 \mathrm{~nm}$ units that aggregate and form a scale invariant structure which is fractal in nature. ${ }^{11}$ The fractal structure persists to a length scale of around $40 \mathrm{~nm}$. It is agreed that the pore system and surfaces are constantly changing within cement paste, hence any data represent a snapshot in time; however, the rate at which the pore structure is changing slows down considerably within a few weeks (longer in the case of pozzolanic materials). Our samples were stored under saturated conditions for 24 months, hence the rate of chemical activity will be minimal. The importance of the saturated conditions is discussed below.

The electrical model for a saturated porous material such as hardened cement paste (or indeed, rock) can be represented, in its simplest form, by two series connected parallel arrangements similar to that shown in Fig. 1 of the paper. One parallel combination will represent the bulk response from the material; the other will represent the response at the electrode-material interface region (socalled electrode polarization). Now, under saturated or near-saturated conditions deconvolution of these two circuits is relatively straightforward as the time constants $R_{\mathrm{p}} C_{\mathrm{p}}$ for each circuit are quite disparate. Fig. 5 in the paper clearly shows that the spur on the right-hand side of the complex plot (which is part of a much larger arc associated with electrode effects) virtually touches the real axis and hence the impedance associated with this element is eliminated at higher frequencies.

Consider the case when the bulk material resistance $R_{\mathrm{p}}$ increases. This will result in an increase in the time constant of the circuit (i.e. $f_{\mathrm{c}}$ decreases), thereby shifting the complex arc associated with the bulk impedance response to the right in the Argand diagram. This arc will begin to merge into the arc due to electrode polarization, and deconvolution of the two arcs now becomes more difficult. Furthermore, under increasing bulk resistance, the frequency at which the change-over point between the two arcs occurs (here denoted $f_{\mathrm{p}}$ ) will decrease, with the curve at this point becoming elevated above the real axis. The change over frequency $f_{\mathrm{p}}$ can easily be determined from the complex plot; however, this may not represent the low-frequency limit at which the arc associated with the bulk material begins. From the diagram shown in Fig. 2 , this frequency may actually be less than $f_{\mathrm{p}}$.

Consider now the data in Table 1 , in which $f_{\mathrm{p}}$ is 


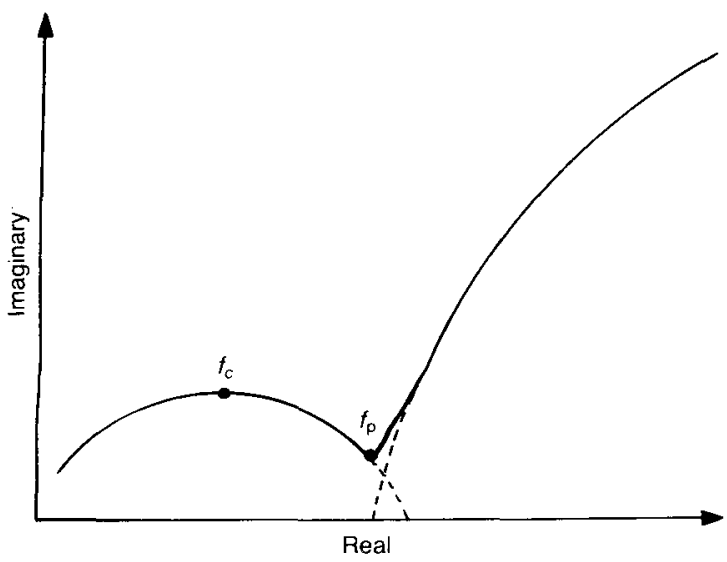

Fig. 2. The complex impedance response of a porous ionic conductor

presented as a function of the gravimetric moisture content of the rock sample. The moisture content varies over the range $0.05 \%-4.3 \%$; when dealing with electrical measurements it is perhaps more informative to use the degree of saturation $S_{\mathrm{r}}$ of the pore space as opposed to the moisture content. If one assumes that the upper value in Table 1 represents a fully saturated sample, then a moisture content of $0.05 \%$ would represent a sample that is virtually bone dry. As $S_{\mathrm{r}}$ for the sample decreases the bulk resistance $R_{\mathrm{p}}$ increases, $f_{\mathrm{c}}$ decreases and the bulk arc will be displaced to the right in the Argand diagram as discussed above. This will result in the turn-over frequency $f_{\mathrm{p}}$ decreasing under decreasing degree of saturation of the sample, as the data presented by $\mathrm{Dr}$ Massey verify.

There is now the problem of the calculated diffusion paths in Table 1 and the apparent anomaly raised by Dr Massey, i.e. that the diffusion path length increases under decreasing saturation. The $D_{0}$ value used by Dr Massey in his calculations is $10^{-9} \mathrm{~m}^{2} / \mathrm{s}$, this value being used at all levels of pore saturation. In our own work a value of $10^{-9} \mathrm{~m}^{2} / \mathrm{s}$ was also used, as this was taken to represent an average diffusion constant of the ionic species within the free capillary water (as opposed to adsorbed or interlayer water). It should be stressed that this represents a diffusion coefficient of the ion species at infinite dilution, and whether this is representative of the diffusion coefficient of the same species in the restricted space of a capillary pore network is open to question (e.g. ionion interactions, influence of surface forces). The value thus represents a first approximation.

The ease with which the water is removed from a porous material will be linked to the distribution of pore sizes within the material, larger pores being depercolated before finer pores. As water is removed from the saturated material, the mobility of the ions (in the free water) will reduce. The diffusion constant at infinite dilution is clearly not applicable in such a situation. Indeed, if one had to invoke a diffusion coefficient under reducing saturation it would be no more than an equivalent or effective diffusion coefficient. This could be several orders of magnitude lower than the value for the ionic species at infinite dilution. For the lowest moisture contents presented in Table 1, all but the very finest pores within the sample would be depercolated. Any ions present would be tightly adsorbed onto the pore surfaces and their mobility reduced, the diffusion coefficient assumed at $4.4 \%$ would not be appropriate at $0.05 \%$; indeed, the bulk polarization mechanism operative at these low levels of saturation could be entirely different from saturated conditions. At low saturation values movement of bound ions in the adsorbed water-phase could be a possible polarization process, as opposed to a diffusion-type process at higher degrees of saturation. If this were the case, then one could not realistically compare the same sample at such extremes of saturation. Consideration of the activation energy of the bulk capacitance (of the saturated material) would also suggest a diffusion-related process. $^{12}$

Care has been taken to present data for saturated conditions. Drying samples will not only result in possible irreversible microstructural changes, but will also reduce ionic mobility within the pore water.

\section{References}

1. Wong P. Z. et al. Surface roughening and the fractal nature of rocks. Phys. Rev. Lett. 1986, 57, No. 5, 637-640.

2. KroHn C. E. Sandstone fractal and euclidean pore volume distributions. J. Geophys. Res., 1988, 93, No. 84, 3286-3296.

3. KROHN C. E. Fractal measurements of sandstones, shales and carbonates. J. Geophys. Res., 1988, 93, No. 84, 3297-3305.

4. KATZ A. J. and Thompson A. H. Fractal sandstone pores: implications for conductivity and pore formation. Phys. Rev. Lett., 1985, 54, No. 12, 1325-1328.

5. Ruffet C. et al. Complex conductivity measurements and fractal nature of porosity. Geophysics, 1991, 56, No. 6, June, 758-768.

6. Wong T. F. et al. Crack aperture statistics and pore space fractal geometry of Westerly granite and Rutland quartz. J. Geophys. Res., 1989,94 , No. 138, pp. 10267-10278.

7. Wong P. Z. Fractal surfaces in porous media. Proc. American Institute of Physics Conf. on Physics and Chemistry of Porous Media (ed. J. R. Banavar et al.), 1987, 2, paper 154, pp. 304-318.

8. WinsLow D. N. The fractal nature of the surface of cement paste Cem. Concr. Res., 1985, 15, No. 5, 817-824.

9. Kriechbaum M. et al. Fractal structure of Portland cement paste during age hardening analysed by small-angle X-ray scattering. Prog. Colloid Polym. Sci., 1989, 79, 101-105.

10. MAGGION R. et al. A scaling approach to the texture of hardened cement pastes. Proc. 9th Int. Congr. on the Chemistry of Cement, New Delhi, 1992, 4, pp. 214-219.

11. Allen A. J. et al. Development of the fine porosity and gel structure of hydrating cement systems. Philos. Mag., 1987, B56, No. 3, 263-288.

12. McCarter W. J. Effects of temperature on conduction and polarization in Portland cement mortar. J. Am. Ceram. Soc., 1995 , 78, No. 2, 411-415. 